\title{
PTEN Mutation Analysis Was Not Performed
}

National Cancer Institute

\section{Source}

National Cancer Institute. PTEN Mutation Analysis Was Not Performed. NCI Thesaurus.

Code C160490.

An indication that PTEN mutation analysis was not performed during the study. 